\title{
Percutaneous endoscopy to diagnose malignancy in gastric outlet obstruction of excluded stomach after gastric bypass
}

\author{
Waseem Ahmada, Joshua Rubinn a,b, Wilson Kwonga,b \\ University of California San Diego, La Jolla, California, USA
}

\begin{abstract}
Gastric cancer in the excluded stomach after Roux-en-Y gastric bypass is a rare finding and most reported diagnoses are made via surgery. Endoscopic access to the excluded stomach is difficult, even with balloon-assisted enteroscopy. We present the case of a 74-year-old woman with malignant gastric outlet obstruction of the excluded stomach, 41 years after Roux-en-Y gastric bypass. Minimally invasive access to the excluded stomach was obtained by placement of a percutaneous gastrostomy tube, followed by insertion of a pediatric gastroscope through the gastrostomy tube tract. This novel approach provides minimally invasive access to the excluded stomach in patients with high suspicion of pathology in the excluded stomach, when balloonassisted enteroscopy is not technically feasible or available.
\end{abstract}

Keywords Gastric adenocarcinoma, excluded stomach, Roux-en-Y gastric bypass, gastrostomy tube, gastroscope

Ann Gastroenterol 2017; 30 (3): 1-3

\section{Introduction}

The development of gastric cancer in the excluded stomach after Roux-en-Y gastric bypass (RYGB) is a rare event, with few reports in the literature [1]. Examination of the excluded stomach via endoscopy is challenging, ultimately requiring most diagnoses to be made through surgery. We describe a novel, minimally invasive method of passing a pediatric gastroscope through a gastrostomy tube tract to diagnose malignancy in the excluded stomach. To our knowledge, this is the first report of such a case.

\section{Case report}

A 74-year-old female who had undergone RYGB in 1975 , with lung cancer in remission and vaginal cancer complicated by rectovaginal fistula requiring colostomy diversion, presented with one month of early satiety, 40-pound weight loss, and left

aDepartment of Medicine (Waseem Ahmad, Joshua Rubin, Wilson Kwong); 'Division of Gastroenterology (Joshua Rubin, Wilson Kwong), University of California San Diego, La Jolla, California, USA

Conflict of Interest: None

Correspondence to: Waseem Ahmad, 200 W. Arbor Drive \#8425, San Diego, CA 92103, USA, e-mail: wlahmad@ucsd.edu

Received 02 January 2017; accepted 06 February 2017; published online 9 March 2017

DOI: https://doi.org/10.20524/aog.2017.0132 upper quadrant abdominal pain. Her physical exam was notable for fullness and tenderness to palpation of the abdominal left upper quadrant. Laboratory testing was notable for a normocytic anemia with hemoglobin of $8.4 \mathrm{~g} / \mathrm{dL}$ and normal iron studies. Her white blood cell count, liver function tests, and lipase were within normal limits. Abdominal computed tomography (CT) revealed severe dilation of the stomach, with thickening of the pylorus and duodenal bulb consistent with gastric outlet obstruction of the excluded stomach (Fig. 1). Per-oral single-balloon enteroscopy was performed and revealed a normal gastric pouch and gastrojejunal anastomosis, but was unable to reach the excluded stomach because of sharp angulations of the small bowel, probably due to abdominal adhesions.

The patient was evaluated by the surgical service, who deemed her a poor surgical candidate for laparoscopic exploration. An ultrasound-guided 12-French (Fr) gastrostomy tube was placed percutaneously by interventional radiology into the excluded stomach for decompression of the gastric outlet obstruction of the excluded stomach. In subsequent months, upsizing of the gastrostomy tube tract was performed until a 24-Fr tube was in place. She then underwent endoscopic evaluation under moderate sedation using a pediatric gastroscope (EVIS EXERA III GIF-XP190N gastroscope) with an outer diameter of $5.4 \mathrm{~mm}$. For this procedure, carbon dioxide was used for insufflation but no trocar was used. The patient's existing 24-Fr gastrostomy tube was deflated and easily removed through the tract. A guidewire was coiled into the excluded stomach under endoscopic guidance. The pediatric gastroscope was advanced alongside the guidewire into the excluded stomach. A large, friable, ulcerated mass (Fig. 2) was identified and multiple biopsies were taken, later identified 


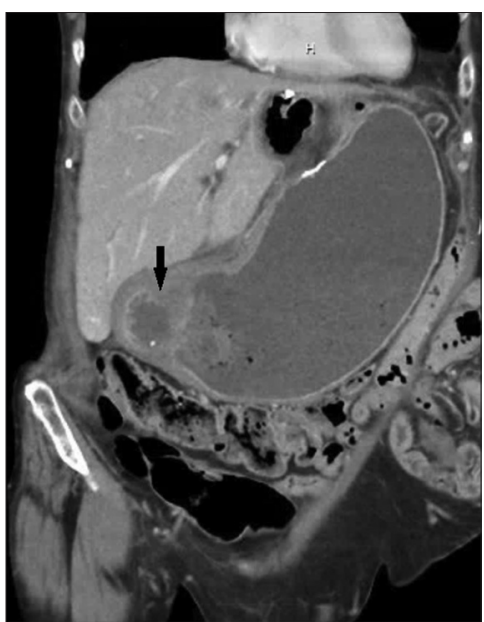

Figure 1 Coronal computed tomography image revealing gastric outlet obstruction of the excluded stomach. Arrow reveals thickening of the gastric wall

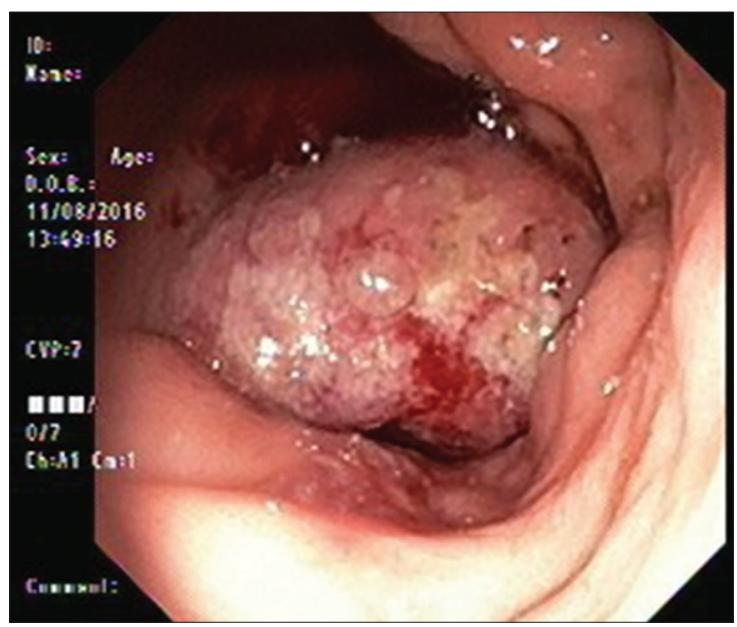

Figure 2 Endoscopic view of a large friable, ulcerated mass within the excluded stomach. A later biopsy proved it to be a poorly differentiated gastric adenocarcinoma

as poorly differentiated adenocarcinoma with signet ring and mucinous features consistent with primary gastrointestinal malignancy. After removal of the gastroscope, a new 24-Fr gastrostomy tube was advanced without difficulty into the excluded tract and its position was confirmed via injection of contrast through the tube. She was deemed a poor surgical and chemotherapy candidate, eventually passing away in a hospice.

\section{Discussion}

Gastric cancer in the excluded stomach following RYGB is rare, with an estimated incidence of $0.03 \%$ [2]. The case presented in the current report represents the longest interval between RYGB and development of gastric cancer in the excluded stomach, at 41 years. Currently there is no clear explanation behind the development of cancer in the excluded stomach after bariatric surgery. Some prominent hypotheses include bile reflux, gastric stasis, chronic reflux, dysplasia due to excess intraluminal pressure and ischemia, and metaplasia due to chronic inflammation [3]. Diagnosis can be challenging, as symptoms are nonspecific and often go unrecognized, resulting in a late presentation. The most frequently reported symptoms include dysphagia and food intolerance, nausea and vomiting, abdominal or epigastric pain, and weight loss [4].

Post-RYGB patients with symptoms that raise concern for gastric malignancy require assessment of the excluded stomach, as non-adenocarcinoma pathologies, such as linitis plastica and gastrointestinal stromal tumors, have been reported [5,6]. Unfortunately, accessing the excluded stomach is challenging because of the altered anatomy following RYGB. Several methods have been developed for both diagnostic and therapeutic interventions, each with limitations (Table 1). Retrograde endoscopic intubation of the excluded stomach via a pediatric colonoscope can be successful in $65 \%$ of patients [7]. However, this method is limited when a longer Roux limb is created. Balloon-assisted enteroscopy has emerged as a useful method of accessing the excluded stomach. Though the total length of the afferent and efferent limbs is less of a concern, major adhesions and technical experience can limit its success. Laparoscopic transgastric endoscopy has also been used as an approach to access the excluded stomach safely, though this can also be more complex in the setting of adhesions [8].

To circumvent some of the challenges posed by the above approaches, a gastrostomy tube can be placed percutaneously by interventional radiology in the excluded stomach, under fluoroscopic, endoscopic ultrasound (EUS), or CT guidance. More recently, an EUS-guided approach by interventional gastroenterologists can be employed [9]. CT- or EUS-guided fine-needle aspiration can be used if the mass in the excluded stomach is seen with these imaging techniques; however, this is often not the case. As in this patient, a percutaneously placed gastrostomy tube can be upsized to 24-Fr to facilitate endoscopy of the excluded stomach; the gastrostomy tract is likely to be mature and stable for percutaneous endoscopy after 3-4 weeks. The gastrostomy tube should be at least 20-Fr or 22-Fr to allow passage of the pediatric gastroscope directly into the stomach. If a smaller tube is used, a controlled radial expansion dilating balloon can be used to dilate the gastrostomy tube tract to 6-8 $\mathrm{mm}$, allowing easy passage of the gastroscope. A guidewire can be placed through the tube into the stomach to maintain access, but this is often not necessary.

This case highlights the importance of endoscopic evaluation of the excluded stomach in RYGB patients with features that raise concern for gastric malignancy, such as unexpected delayed weight loss, worsening anemia, and gastric outlet obstruction. In these RYGB patients with worrisome symptoms, we demonstrate the feasibility of a minimally invasive percutaneous endoscopic approach, using a gastrostomy tube for endoscopic access to the excluded stomach, when balloon-assisted enteroscopy is not technically feasible or is unavailable. 
Table $1 \mathrm{~A}$ list of methods that can be used to access the excluded stomach and common limitations that may discourage their use

\begin{tabular}{|c|c|}
\hline Method & Major limitations \\
\hline Push enteroscopy & $\begin{array}{l}\text { Can be technically challenging } \\
\text { Limited by presence of longer Roux limb } \\
\text { Limited success rates } \\
\text { Tedious }\end{array}$ \\
\hline Single or double balloon enteroscopy & $\begin{array}{l}\text { Can be technically challenging } \\
\text { May require significant technical experience } \\
\text { Limited by access } \\
\text { Limited by presence of adhesions }\end{array}$ \\
\hline Laparoscopic transgastric endoscopy & Difficult procedure in the setting of adhesions \\
\hline CT-FNA & Can only be performed if a large mass is seen on CT \\
\hline EUS-FNA & Can only be performed if mass seen on EUS \\
\hline Creation of gastric-gastric fistula using Axios stent & Significant risk for perforating excluded stomach and spreading tumor tissue \\
\hline Surgery (laparoscopy, laparotomy) & $\begin{array}{l}\text { Can be technically challenging because of adhesions and altered anatomy } \\
\text { Carries surgical procedure risks }\end{array}$ \\
\hline Percutaneous endoscopy via gastrostomy tube & $\begin{array}{l}\text { Inability to distend remnant stomach with air } \\
\text { Requires serial dilation to facilitate endoscopy } \\
\text { Challenging if surrounded by loops of small bowel and colon }\end{array}$ \\
\hline
\end{tabular}

\section{References}

1. Raghavendra RS, Kini D. Benign, premalignant, and malignant lesions encountered in bariatric surgery. JSLS 2012;16:360-372.

2. Tinoco A, Gottardi LF, Boechat ED. Gastric cancer in the excluded stomach 10 years after gastric bypass. Case Rep Surg 2015;2015:468293.

3. Kuruba R, Jawad M, Karl RC, Murr MM. Technique of resection of esophageal adenocarcinoma after Roux-en-Y gastric bypass and literature review of esophagogastric tumors after bariatric procedures. Surg Obes Relat Dis 2009;5:576-581.

4. Scozzari G, Trapani R, Toppino M, Morino M. Esophagogastric cancer after bariatric surgery: systematic review of the literature. Surg Obes Relat Dis 2013;9:133-142.

5. Haenen FW, Gys B, Moreels T, Michielsen M, Gys T, Lafullarde T. Linitis plastica of the bypassed stomach 7 years after Roux-en-Y gastric bypass. A case report. Acta Chir Belg 2016 [Epub ahead of print].

6. AbellanAbellán I, Ruíz de Angulo D, Parrilla P. Incidental gastric gastrointestinal stromal tumor (GIST) in the excluded stomach after Roux- en-Y gastric bypass: a case report and review of the literature. Surg Obes Relat Dis 2014;10:e13-e14.

7. Sinar DR, Flickinger EG, Park HK, Sloss RR. Retrograde endoscopy of the bypassed stomach segment after gastric bypass surgery: unexpected lesions. South Med J 1985;78:255-258.

8. Ceppa FA, Gagné DJ, Papasavas PK, Caushaj PF. Laparoscopic transgastric endoscopy after Roux-en-Y gastric bypass. Surg Obes Relat Dis 2007;3:21-24.

9. Attam R, Leslie D, Freeman M, Ikramuddin S, Andrade R. EUSassisted, fluoroscopically guided gastrostomy tube placement in patients with Roux-en-Y gastric bypass: a novel technique for access to the gastric remnant. Gastrointest Endosc 2011;74:677-682. 